\title{
Сенсор электрического поля на основе двойной квантовой точки в микрорезонаторе
}

\author{
(C) А.В. Цуканов ${ }^{1,2}$, В.Г. Чекмачев ${ }^{1,2,9}$ \\ ${ }^{1}$ Московский фризико-технический институт, \\ 141701 Догопрудный, Россия \\ ${ }^{2}$ Физико-технологический институт Российской академии наук, \\ 117218 Москва, Россия \\ ` E-mail: vgchekmachev@mail.ru
}

(Получена 1 февраля 2017 г. Принята к печати 22 фревраля 2017 г.)

\begin{abstract}
Приведена схема оптического квантового сенсора внешнего электрического поля на основе двойной квантовой точки, помещенной в высокодобротный полупроводниковый микрорезистор. Разработана модель динамических процессов, происходящих в данной системе, исследованы ее спектральные характеристики, а также проведено изучение шумовой устойчивости сенсора. Показано, что благодаря особенностям дизайна подобное устройство обладает некоторыми преимуществами, такими, как высокая чувствительность, наличие различных каналов для возбуждения и измерения, возможность точного определения пространственного распределения поля.
\end{abstract}

DOI: $10.21883 /$ FTP.2017.09.44890.8485

\section{1. Введение}

Одиночные полупроводниковые квантовые точки (КТ) обладают уникальными оптическими и фотофизическими свойствами. Эти объекты уже были использованы при создании прототипов ряда устройств: генераторов одиночных фотонов, оптоэлектронных приборов, фотокатализаторов и др. [1]. Возможно получение и упорядоченных массивов двойных квантовых точек (ДКТ) [2]. Интегрирование КТ в высокодобротные оптические микрорезонаторы позволяет использовать эти наноструктуры во многих перспективных областях современной науки, например в квантовой информатике в роли элементарного носителя информации - кубита [3-6]. Технология создания таких структур состоит из нескольких этапов. Вначале на подложке из InAs методом молекулярно-лучевой эпитаксии (МЛЭ) формируется нижняя часть структуры. Затем на ее поверхность наносятся маркеры для образования массива наноотверстий, в которые помещается GaAs, выступающий в качестве зародышей для роста КТ. Сверху напыляется еще три слоя InAs с вкраплениями GaAs, что позволяет улучшить оптические свойства системы. Решетка вертикальных отверстий фотонного кристалла (ФК), формируемых литографически, приводит к дискретизации фотонного спектра в структуре и способствует локализации электромагнитного поля в ее центральной области с несколькими пропущенными отверстиями (дефектами решетки). Таким образом КТ могут оказаться в пучностях собственной моды оптического микрорезонатора (МР). Кроме того, отдельно взятые КТ взаимодействуют с внешним лазерным полем, частота которого (как и частота моды МР) выбирается близкой к частоте электронного перехода в КТ (несколько сотен мэВ).

Перейдем к обзору экспериментальных исследований гибридных объектов, включающих в себя КТ, МР и лазер. В работе [7] авторы изучили когерентное взаимодействие мощного лазерного излучения и поляритонов в системе „КТ + МР“. Образец для исследования был получен с помощью метода МЛЭ и состоял из жертвенного слоя $\mathrm{Al}_{0.8} \mathrm{Ga}_{0.2} \mathrm{As}$ толщиной $\sim 900$ нм, со следующим за ним слоем GaAs толщиной $\sim 145$ нм, содержащим монослой InAs KT. В результате процесса выращивания структуры плотность КТ составила 60-80 мкм ${ }^{-2}$. Затем при помощи электронно-лучевой литографии с последующим реактивным ионным травлением и удалением вспомогательного слоя формируется ФК с постоянной решетки $a=246$ нм и радиусом отверстий $r \sim 60$ нм. Функции МР выполняет линейный дефект решетки ФК в виде трех отверстий. Для улучшения добротности отверстия, прилегающие к МР, были слегка смещены. Наблюдение электрон-фотонных осцилляций Раби в данной системе является подтверждением когерентного взаимодействия между лазером, МР и КТ. Этот эффект может быть использован для выполнения однои двухкубитных вентилей в квантовом компьютере [8]. Однако и некогерентное взаимодействие электрона с квантовыми полями, когда скорости диссипативных процессов оказываются много больше частоты Раби, дает возможность реализовать устройства, принцип работы которых базируется на анализе спектроскопического отклика системы (например, сенсоры и детекторы внешних полей).

В данной работе предлагается схема сенсора внешнего электрического поля на основе ДКТ. Нами разработана математическая модель, позволяющая описать квантово-механическую эволюцию системы, состоящей из одного электрона, находящегося в ДКТ, при взаимодействии с модой МР и полем лазера. Для решения этой задачи используется подход, базирующийся на основе уравнения Линдблада для матрицы плотности электронфотонной системы с учетом ухода фотонов из резонато- 
ра в континуум и релаксации возбужденного электрона с испусканием фотона или фонона. Будут исследованы основные характеристики прибора, такие как рабочий диапазон электрических полей, чувствительность, соотношение сигнал/шум, проведен сравнительный анализ с существующими аналогами. Стоит отметить, что одной из важных особенностей нашей концепции является использование асимметричных ДКТ, энергии перехода между основными и возбужденными состояниями которых в левой КТ и правой КТ отличаются. Также за счет

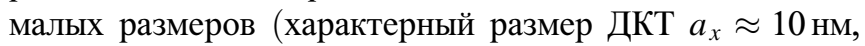
$a_{y} \approx 5$ нм) появляется возможность измерять пространственное распределение электрического поля с высокой точностью. Что же касается диссипативных эффектов, то основными каналами потери когерентности являются процессы, связанные с уходом фотонов из МР. Существующие полупроводниковые МР, как правило, имеют добротность $Q \sim 10^{4}-10^{6}$. Будет показано, что этого достаточно для использования ДКТ в роли сенсора внешнего электрического поля.

\section{2. Модель и основные уравнения}

На рис. 1, а представлена принципиальная схема прибора. Устройство состоит из полупроводниковой одноэлектронной ДКТ [9], сформированной КТ $A$ и КТ $B$ и
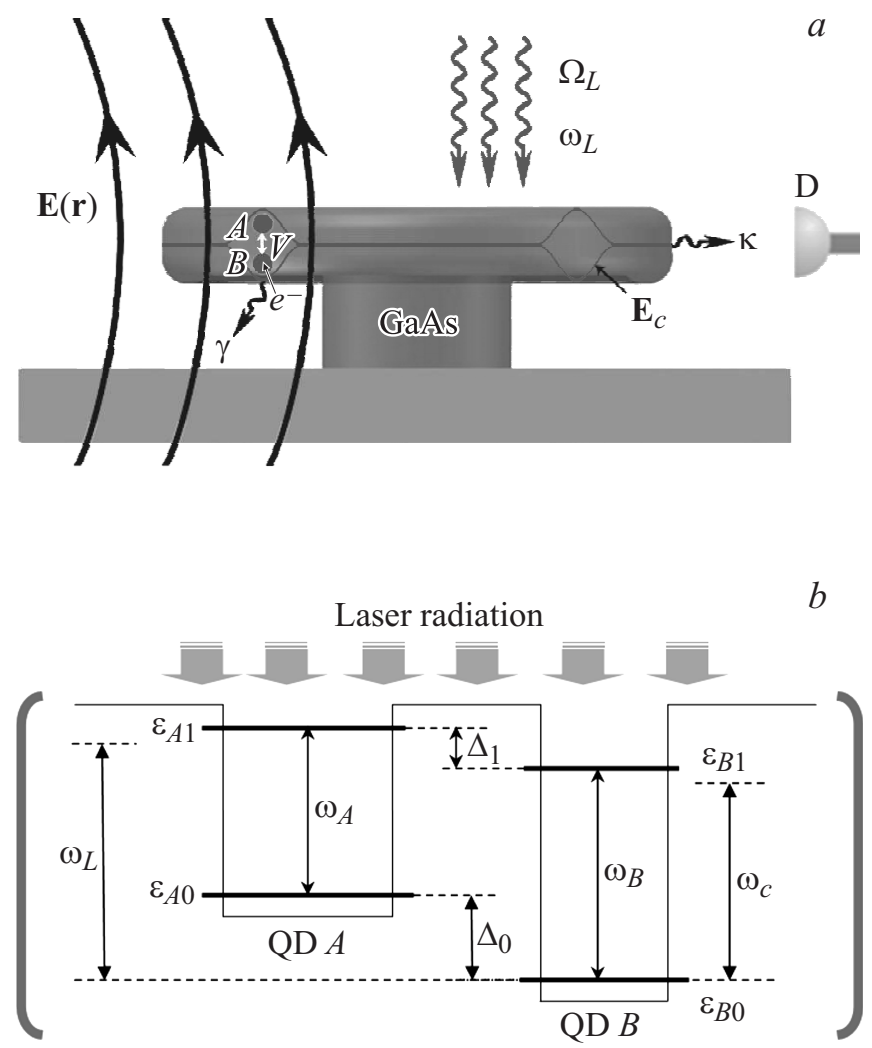

Рис. 1. $a-$ схема сенсора на основе ДКТ, микрорезонатора и лазера. $b-$ схема двойной квантовой точки в комбинированном поле лазера и резонатора. помещенной в высокодобротный МР, который выполнен в виде микродиска на основе GaAs, изготовленного по ранее апробированной технологии [2]. ДКТ расположена в краевой области МР, где напряженность электрического поля его оптической моды $\mathbf{E}_{c}$ максимальна. На систему „ДКТ + MP“ $^{\star}$ наложено лазерное поле с частотой $\omega_{L}$ и амплитудой $\Omega_{L}$. Система помещена во внешнее электрическое поле $\mathbf{E}(\mathbf{r})$, параметры которого подлежат определению. В результате его воздействия на ДКТ имеет место изменение расщепления возбужденных уровней ДКТ за счет эффекта Штарка [10]. Вследствие этого происходит перераспределение заселенности состояний системы, пространство которых включает все возможные сочетания $\{|i, n\rangle\}$, где $i=[A 0, B 0, A 1, B 1]-$ уровни ДКТ, а $n \in[0,1,2, \ldots]$ - количество фотонов, находящихся в МР. При взаимодействии лазерного классического (многофотонного) поля и квантового (однофотонного) поля МР с ДКТ происходят переходы электрона между ее уровнями. Возбужденный электрон может туннелировать из КТ $A$ в КТ $B$, а коэффициент туннелирования равен $V$. В результате состояния изолированных КТ гибридизируются, вследствие чего появляются два новых транспортных уровня $|+\rangle$ и $|-\rangle$. Их роль в эволюции системы будет описана в разд. 3 данной работы. При переходе с возбужденного уровня ДКТ на основной в моду МР испускается фотон, тогда как при обратном переходе фотон поглощается. Для функционирования прибора необходимо, чтобы вероятность нахождения системы в состояниях с ненулевым числом фотонов в МР была достаточно высокой, так как именно излучение от MP измеряется детектором D. В ходе эксперимента проводится сканирование частоты лазерного поля для поиска той, при которой интенсивность излучения, попадающего на датчик, будет максимальна. Затем результат сверяется с эталонной кривой, после чего рассчитывается амплитуда измеряемого поля $|\mathbf{E}(\mathbf{r})|$.

Обсудим применимость четырехуровневого приближения для описания электронного спектра ДКТ. Одиночная полупроводниковая КТ представляет собой, как правило, кристаллит-многогранник с высокой симметрией. Ее возбужденные состояния (аналоги атомных p-орбиталей) ортогональны друг другу и ориентированы вдоль соответствующих декартовых осей. Это задает правила отбора для оптических переходов в КТ, связывающих основное и одно из возбужденных состояний. Например, если внешнее поле поляризовано вдоль оси $x$, то будут возбуждаться только переходы между основным состоянием и $p_{x}$-состоянием (поляризационная селективность). Если центры КТ лежат на оси $x$, то приведенные рассуждения остаются справедливыми и для ДКТ. При этом гибридизируются только $p_{x}$-состояния двух КТ ( в наших обозначениях $-A_{1}$ и $B 1)$. Электронная гибридизация приводит к смещению энергий этих (возможно, первоначально вырожденных) состояний на величину порядка энергии туннелирования $V(0.5-0.1$ мэВ при расстоянии между КТ 5-10 нм). Это обеспечивает дополнительную изоляцию рабочих 
уровней схемы от прочих, что может быть важно при наличии небольшой структурной асимметрии, приводящей к остаточному перемешиванию $p$-состояний (частотная селективность). Влияние состояний электронного континуума, лежащих вблизи края потенциального барьера ДКТ, оказывается незначительным вследствие малости амплитуды их волновых функций, делокализованных по всему объему МР.

Потенциальный профиль структуры в направлении роста и схема ее энергетических уровней показаны на рис. $1, b$. Гамильтониан сформированной таким образом четырехуровневой одноэлектронной ДКТ и МР имеет вид

$$
\begin{aligned}
H_{0}= & \omega_{c} a^{+} a+\varepsilon_{A 0}|A 0\rangle\left\langle A 0\left|+\varepsilon_{B 0}\right| B 0\right\rangle\left\langle B 0\left|+\varepsilon_{A 1}\right| A 1\right\rangle\langle A 1| \\
& +\varepsilon_{B 1}|B 1\rangle\langle B 1|-V(|A 1\rangle\langle B 1|+| B 1\rangle\langle A 1|)
\end{aligned}
$$

где $\omega_{c}$ - частота моды MP, $a$ - оператор уничтожения фотона в данной моде, $\varepsilon_{A 0}, \varepsilon_{B 0}, \varepsilon_{A 1}, \varepsilon_{B 1}-$ энергии уровней изолированных КТ, $V>0-$ матричный элемент (энергия) туннелирования электрона между возбужденными уровнями КТ (здесь и далее полагаем постоянную Планка равной единице). Поле МР вызывает вертикальные переходы $|A 0\rangle \leftrightarrow|A 1\rangle$ и $|B 0\rangle \leftrightarrow|B 1\rangle$ между основными $(|A 0\rangle$ и $|B 0\rangle)$ и возбужденными $(|A 1\rangle$ и $|B 1\rangle)$ состояниями КТ, которые с учетом приближения вращающейся волны и дипольного приближения описываются гамильтонианом Джейнса-Каммингса:

$$
\begin{aligned}
H_{\mathrm{JC}}= & g_{A}\left(|A 0\rangle\left\langle A 1\left|a^{+}+\right| A 1\right\rangle\langle A 0| a\right) \\
& +g_{B}\left(|B 0\rangle\left\langle B 1\left|a^{+}+\right| B 1\right\rangle\langle B 0| a\right),
\end{aligned}
$$

где $g_{A}$ и $g_{B}-$ не зависящие от времени коэффициенты взаимодействия ДКТ и МР (частоты Раби). Частота электронного перехода в КТ $A(B)$ равна разности энергий ее состояний: $\omega_{A(B)}=\varepsilon_{A(B) 1}-\varepsilon_{A(B) 0}$. Кроме квантового поля МР, ДКТ аналогичным образом взаимодействует еще и с лазерным (классическим) полем, задаваемым гамильтонианом

$$
\begin{aligned}
H_{L}= & \Omega_{A} \cos \left(\omega_{L} t\right)(|A 1\rangle\langle A 0|+| A 0\rangle\langle A 1|) \\
& +\Omega_{B} \cos \left(\omega_{L} t\right)(|B 1\rangle\langle B 0|+| B 0\rangle\langle B 1|),
\end{aligned}
$$

где $\Omega_{A}$ и $\Omega_{B}$ - не зависящие от времени амплитуды (частоты Раби) лазера в КТ $A$ и $B$, а $\omega_{L}$ - частота лазера. Корректность такого описания взаимодействия КТ и лазера была подтверждена экспериментально (см. [4-6]). Таким образом, полный гамильтониан ДКТ в квантовом и классическом полях представляется в виде суммы выражений (1), (2) и (3):

$$
H=H_{0}+H_{\mathrm{JC}}+H_{L} .
$$

Отметим, что в модели (4) мы пренебрегаем туннелированием между основными состояниями КТ, а также диагональными переходами $|A 0\rangle \leftrightarrow|B 1\rangle$ и $|B 0\rangle \leftrightarrow|A 1\rangle$.
Для дальнейшей работы с выражением (4) удобно перейти в систему отсчета, связанную с лазером, с помощью унитарного преобразования $T=\exp \left[-i \omega_{L}(|A 1\rangle\langle A 1|\right.$ $\left.\left.+|B 1\rangle\langle B 1|+a^{+} a\right) t\right]$. При этом гамильтониан (4) преобразуется как

$$
\tilde{H}=T^{\dagger} H T+i \frac{\partial T^{\dagger}}{\partial t} T=\tilde{H}_{0}+\tilde{H}_{\mathrm{jC}}+\tilde{H}_{L},
$$

где

$$
\begin{aligned}
& \tilde{H}_{0}=\Delta a^{+} a-\frac{1}{2}\left(\Delta_{1}+\delta_{A}-\delta_{B}\right)(|A 0\rangle\langle A 0|-| B 0\rangle\langle B 0|) \\
& +\frac{1}{2}\left(\delta_{A}+\delta_{B}-\Delta_{1}\right)|A 1\rangle\left\langle A 1\left|+\frac{1}{2}\left(\delta_{A}+\delta_{B}+\Delta_{1}\right)\right| B 1\right\rangle\langle B 1| \\
& -V(|A 1\rangle\langle B 1|+| B 1\rangle\langle A 1|), \\
& \tilde{H}_{L}=\frac{\Omega_{A}}{2}(|A 1\rangle\langle A 0|+| A 0\rangle\langle A 1|) \\
& \quad+\frac{\Omega_{B}}{2}(|B 1\rangle\langle B 0|+| B 0\rangle\langle B 1|), \quad \tilde{H}_{\mathrm{JC}}=H_{\mathrm{JC}}
\end{aligned}
$$

и введены следующие обозначения: $\Delta_{0(1)}=\varepsilon_{B 0(1)}$ $-\varepsilon_{A 0(1)}-$ разность энергий основных (возбужденных) состояний КТ $A$ и КТ $B, \delta_{A(B)}=\omega_{A(B)}-\omega_{L}-$ отстройка частот перехода в КТ $A(B)$ и лазера, $\Delta_{A(B)}=\omega_{c}-\omega_{A(B)}-$ отстройка частот МР и перехода в КТ $A(B)$ и $\Delta=\omega_{c}-\omega_{L}-$ отстройка частот МР и лазера. Обращаем внимание на то, что в новой системе отсчета гамильтониан не зависит от времени.

Помимо взаимодействия с монохроматическими полями, МР и ДКТ контактируют с континуумом (резервуаром) фотонных и фононных мод, что приводит к потере когерентности электрон-фотонного состояния. Как правило, резервуар общего вида описывается ансамблем гармонических осцилляторов, линейно взаимодействующих с квантовой системой в приближении Борна-Маркова. Для учета некогерентных процессов мы воспользуемся известным подходом, который базируется на введении так называемых супероператоров Линдблада, описывающих распад фотонных состояний МР и электронных состояний ДКТ. Выпишем уравнение Линдблада для редуцированного оператора $\rho$ плотности ДКТ и МР:

$$
\frac{\partial \rho}{\partial t}=-i[\tilde{H}, \rho]+L_{\Sigma} \rho
$$

где $L_{\Sigma} \rho=\sum_{m} \gamma_{m} L_{m} \rho-$ суммарный оператор Линдблада, a $L_{m} \rho=2 A_{m} \rho A_{m}^{\dagger}-A_{m}^{\dagger} A_{m} \rho-\rho A_{m}^{\dagger} A_{m}-$ парциальный оператор Линдблада, характеризующий распад некоторой величины, которой соответствует оператор $A_{m}\left(\gamma_{m}-\right.$ скорость распада). Нас будут интересовать заселенности состояний электрон-фотонной системы, которые равны диагональным элементам матрицы плотности $\rho$. В данной работе мы ограничимся рассмотрением диссипации фотонов из МР $(m=1)$ со скоростью $\kappa$ и распадом возбужденных состояния КТ $A(m=2)$ и $B(m=3)$, 
обусловленным испусканием фотона или оптического фонона со скоростями $\gamma_{A}$ и $\gamma_{B}$ соответственно:

$$
\begin{gathered}
A_{1}=a, \quad \gamma_{1}=\kappa ; \quad A_{2}=|A 0\rangle\langle A 1|, \quad \gamma_{2}=\gamma_{A} ; \\
A_{3}=|B 0\rangle\langle B 1|, \quad \gamma_{3}=\gamma_{B} .
\end{gathered}
$$

Воспользовавшись результатами данного раздела, мы проведем анализ поведения диссипативной системы „ДКТ + МР + лазер“ для реализации сенсора внешнего электрического поля.

\section{3. Спектроскопия}

Для анализа характеристик сенсора на основе ДКТ необходимо получить зависимость частоты лазерного излучения от величины расщепления между возбужденными уровнями. Для этого, пользуясь моделью, приведенной в разд. 2, получим спектроскопический отклик системы для выбранного набора параметров. Результат вычислений представлен на рис. 2, $a$. Далее для удобства все величины будут указаны в относительных единицах, прямо пропорционально частоте MP $\omega_{c}$. При исследовании характеристик устройства здесь и далее используется следующая конфигурация системы „ДКТ + МР + лазер“. Основные уровни КТ $A$ и КТ $B$ имеют отстройку по частоте $\Delta_{0}=-10^{-4}$, что позволяет говорить о возникновении асимметрии в системе. Это свойство будет использовано для получения важного преимущества нашего дизайна сенсора внешнего электрического поля - наличия различных каналов для возбуждения и измерения. Частота резонатора $\omega_{c}$ совпадает с частотой перехода $|B 0\rangle \rightarrow|-\rangle$ при $\Delta_{1}=0$, что позволяет достичь достаточно высокой заселенности состояний системы с количеством фотонов в резонаторе более нуля $\sum P_{\text {exc, } n \geq 1}$. Именно эта величина находится в прямой зависимости с другой важной характеристикой прибора, а именно с соотношением сигнал/шум, которое подробно исследовано в разд. 4 данной работы. Частота лазера $\omega_{L}$ в ходе эксперимента может варьироваться в широком диапазоне $-40 \cdot 10^{-4}+\omega_{c} \leq \omega_{L} \leq 20 \cdot 10^{-4}+\omega_{c}$, что позволяет обнаружить характерные особенности, свойственные динамике гибридной системы на основе ДКТ, МР и лазера. Коэффициент туннелирования электрона между КТ $V=10^{-3}\left(V \gg g_{A, B}, \Omega_{A, B}\right)$ и подобран таким образом, чтобы энергии гибридизированных уровней $|+\rangle$ и $|-\rangle$ попадали в область значений, удовлетворяющих условию наличия в системе строго двухфотонного резонанса, где вероятность $\sum P_{\mathrm{exc}, n \geq 1}$ достигает наибольших значений. Постоянные связи с MP $g_{A, B}=10^{-5}$ и лазером $\Omega_{A, B}=10^{-6}$, удовлетворяют условию $g_{A, B} \gg \Omega_{A, B}$, что приводит к малому уширению резонансных пиков и соответственно позволит повысить шумовую устойчивость прибора (см. разд. 4).

Произведем численную оценку параметров системы. Для частоты MP $\omega_{c} \sim 10^{14}$ Гц, что соответствует $\omega_{A, B} \sim 0.1$ эВ, переменные $\delta$ и $\Delta_{1}$ варьируются в диапазоне $-4 \cdot 10^{11} \Gamma ц \leq \delta \leq 2 \cdot 10^{11} \Gamma ц \quad$ и
$-5 \cdot 10^{11} \Gamma ц \leq \Delta_{1} \leq 5 \cdot 10^{11}$ Гц. Скорость туннелирования электрона между КТ $A$ и КТ $B V=0.1$ эВ. Коэффициенты связи лазера и МР с электроном равны $g_{A, B}=10^{9}$ Гц и $\Omega_{A, B}=10^{8}$ Гц соответственно. Скорость диссипации фотонов, связанная с неидеальностью резонатора, полагается равной $\kappa=10^{8}$ Гц, что соответствует добротности МР $Q=10^{6}$. Данными характеристиками обладает МР, выполненный в виде микродиска и исследованный в работе [11]. Спонтанные переходы между возбужденными и основными уровнями КТ происходят со скоростью $\gamma=10^{7}$ Гц, что может быть реализовано в GaAs KT при гелиевых температурах. Длительность лазерного импульса составляет $T_{c} \sim 1$ нс и подобрана таким образом, чтобы $T_{L} \geq \max \left\{\kappa^{-1}, \gamma^{-1}\right\}$, что позволяет с уверенностью говорить о работе в установившемся режиме.

Создание надежного источника когерентного излучения в необходимом нам диапазоне долгое время представляло собой серьезную проблему. Авторам работы [12] удалось создать терагерцевый квантовый каскадный лазер, обладающий требуемыми оптическими характеристиками. Его активным элементом является гетероструктура InAs/ $\mathrm{AlAs}_{0.16} \mathrm{Sb}_{0.84}$, выращиваемая с помощью МЛЭ, в ходе которой удается получать области толщиной до одного монослоя. Активная среда лазера изготовлена на основе InAs, в котором эффективная масса электрона достигает $m^{*}=0.023 m_{0}$, что в 3 раза меньше эффективной массы в GaAs. Подобное свойство InAs позволяет добиться оптического усиления в 5 раз больше, чем в GaAs. Вторым важным компонентом структуры является барьер из $\mathrm{AlAs}_{0.16} \mathrm{Sb}_{0.84}$, который обладает крайне высоким смещением зоны проводимости, достигающей $E_{C B O}=2.21$ эВ. Также существенной особенностью является то, что подобные лазеры обладают достаточно компактными размерами порядка одного миллиметра, а рабочая температура может доходить до $200 \mathrm{~K}$. Частотный диапазон такого лазера находится в пределах от 1.2 до 5.2 ТГц.

Проанализируем полученный спектроскопический отклик системы. На рис. 2, a видны две характерные гиперболы. Верхняя отвечает наличию в системе резонанса лазерного поля с переходом между основным и нижним гибридизированным уровнем в КТ $A$ (переход $|A 0\rangle \rightarrow|+\rangle)$, т. е. выполняется равенство $\omega_{L}=\Delta_{A}-\sqrt{V^{2}+\left(\Delta_{1} / 2\right)^{2}}-\Delta_{1} / 2$. Нижняя ветвь удовлетворяет условию резонанса лазерного поля с верхним гибридизированным уровнем КТ $A$ (переход $|A 0\rangle \rightarrow|-\rangle$ ), тогда частота лазера $\omega_{L}=\Delta_{A}+\sqrt{V^{2}+\left(\Delta_{1} / 2\right)^{2}}+\Delta_{1} / 2$. Варьируя частоту лазерного импульса, становится возможным найти те значения параметров $\delta$ и $\Delta_{1}$, при которых вероятность возбуждения в состояния, имеющие один и более фотонов $\left(\sum P_{\mathrm{exc}, n \geq 1}\right)$ в резонаторе, максимальна. Эта зона обозначена на рис. 2, $a$ черным штрихованным квадратом, а на рис. $2, b$ представлена детальная картина спектроскопического отклика системы в данной области. 

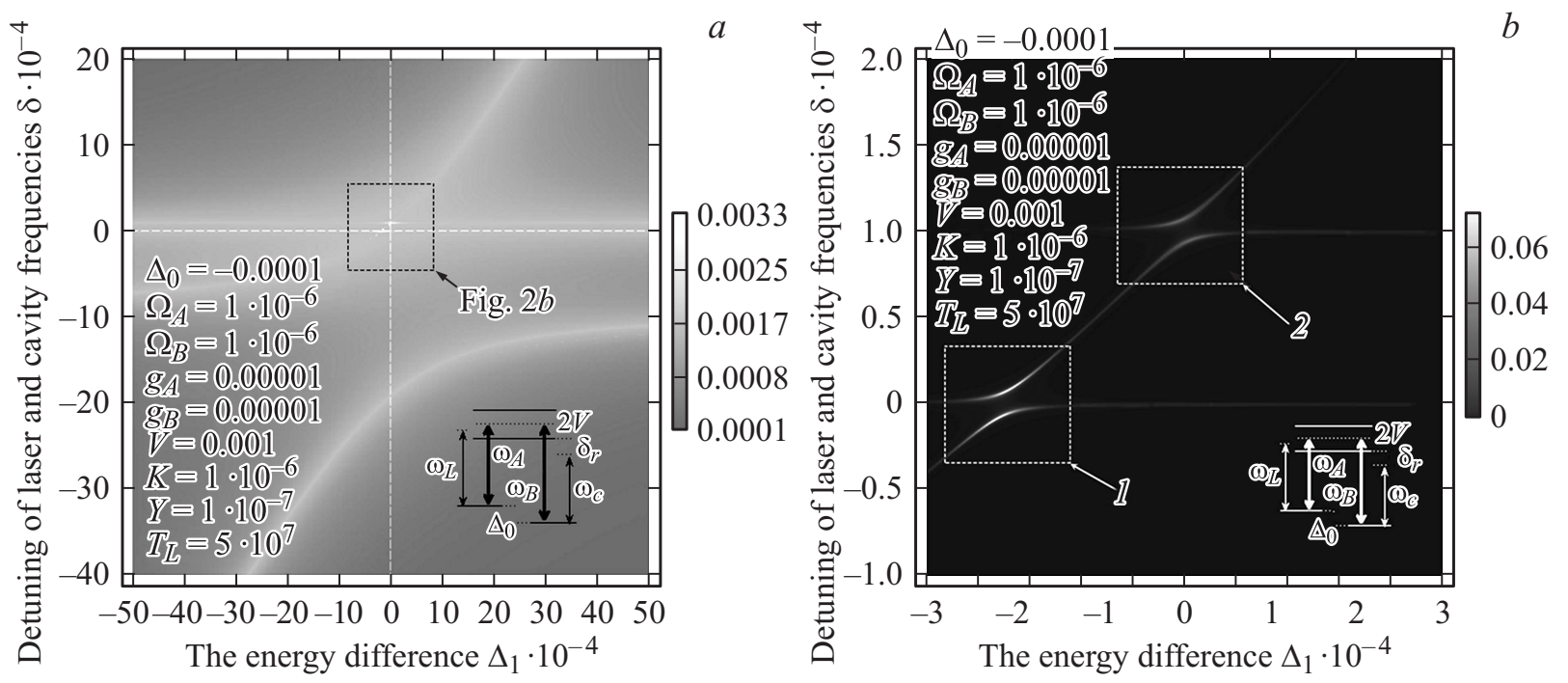

Рис. 2. $a-$ спектроскопический отклик системы $\left(\delta-\right.$ разность частот резонатора и лазера, $\Delta_{1}-$ разность энергий возбужденных уровней КТ $A$ и КТ $B$ ). В нижнем левом углу приведены параметры системы, для которых производился расчет. В нижнем правом углу представлена схема уровней ДКТ. Справа расположена шкала, соответствующая заселенности состояний с количеством фотоном в МР больше либо равном одному. Черный штрихованный квадрат - область, детализированная на рис. $2, b$. $b$ - спектроскопический отклик системы. Шкала справа соответствует заселенности состояний $\sum P_{\text {exc, } n \geq 1}$. Белые штрихованные квадраты - области характерных антипересечений 1 и 2.

Рассмотрим более подробно полученный результат. На рис. 2, $b$ видны два характерных антипересечения. Первое указывает на подключение к резонансу с модой МР второй двухуровневой системы, формируемой состояниями $|A 0\rangle$ и $|+\rangle$. В данной окрестности частоты лазера, МР и гибридизированных уровней ДКТ практически совпадают, что делает возможным закачку фотонов лазерного поля в МР через полупроводниковую структуру. В связи с этим вблизи этого антипересечения наблюдается высокая вероятность заселения уровней с количеством фотонов $n \geq 1$, а максимальное значение достигает величины $\sum P_{\mathrm{exc}, n \geq 1}=0.08$. Второе антипересечение возникает в области совпадения параметров системы с условием строгого двухфотонного резонанса, а именно $\Delta_{B}=\omega_{c}-\omega_{B}=-V, \Delta_{0}=-\delta(9)$. Это значит, что заселенность транспортных состояний $|A 1,0\rangle$ и $|B 1,0\rangle$ в окрестности $\delta=1 \cdot 10^{-4}, \Delta_{1}=0$ невелика, что позволяет получить достаточно высокую заселенность состояний $|A 0, n\rangle,|B 0, n\rangle,|A 1, n\rangle,|B 1, n\rangle ; n \geq 1$. Максимальная вероятность возбуждения в состояния с количеством фотонов в резонаторе больше нуля здесь достигает значения $\sum P_{\mathrm{exc}, n \geq 1}=0.04$.

\section{4. Результаты}

Для определения основных характеристик сенсора произведем более детальное изучение спектроскопического отклика гибридной системы „ДКТ + МР“ на воздействие внешнего лазерного поля. Наша задача произвести подбор параметров, которые были бы пригодны для реализации устройства. Необходимо выделить то место на графике, где при малом изменении величины электрического поля, связанной с расщеплением $\Delta_{1}$, отстройка частот лазера и резонатора будет изменяться на максимальную величину. Как будет показано далее, именно эта характеристика напрямую связана с чувствительностью исследуемого прибора. При этом, однако, следует учесть, что интенсивность получаемого сигнала, представленная величиной $\sum P_{\text {exc, } n \geq 1}$, должна быть много больше величины шума, равной уширению резонансного контура на высоте $\max \left\{\sum P_{\mathrm{exc}, n \geq 1}\right\} / 2$.

Всем этим условиям соответствуют два участка на рис. 2, $b$, а именно $2 \cdot 10^{-4} \leq \delta \leq-1 \cdot 10^{-4}$, $0 \leq \Delta_{1} \leq 0.5 \cdot 10^{-4}$ и $0 \leq \delta \leq 2 \cdot 10^{-4}, 1 \cdot 10^{-4} \leq \Delta_{1} \leq$ $\leq 2 \cdot 10^{-4}$, и, как было отмечено в разд. 3 , максимальная вероятность возбуждения $P_{\max }=\max \left\{\sum P_{\mathrm{exc}, n \geq 1}\right\}$ больше в первой области, чем во второй. Однако вблизи первого антипересечения, за счет того что частоты лазера и МР практически равны $(\delta \approx 0)$, пропадает селективность системы по частоте. Следовательно, для функционирования устройства пригоден только второй участок, а на рис. 3, $a$ представлена детальная картина спектроскопического отклика системы для этого набора параметров. Наличие различных каналов для возбуждения и измерения является одним из конструктивных преимуществ сенсора на основе ДКТ.

В работе [13] приведена конструкция оптического наноэлектрометра на основе полупроводниковой гетероструктуры и исследованы его характеристики. Схема экспериментальной установки состоит из одиночной одноэлектронной КТ, изготовленной на основе GaAs и $\mathrm{AlGaAs}$, помещенной в диод Шоттки. Для измерения напряженности электрического поля, наведенного на КТ за 

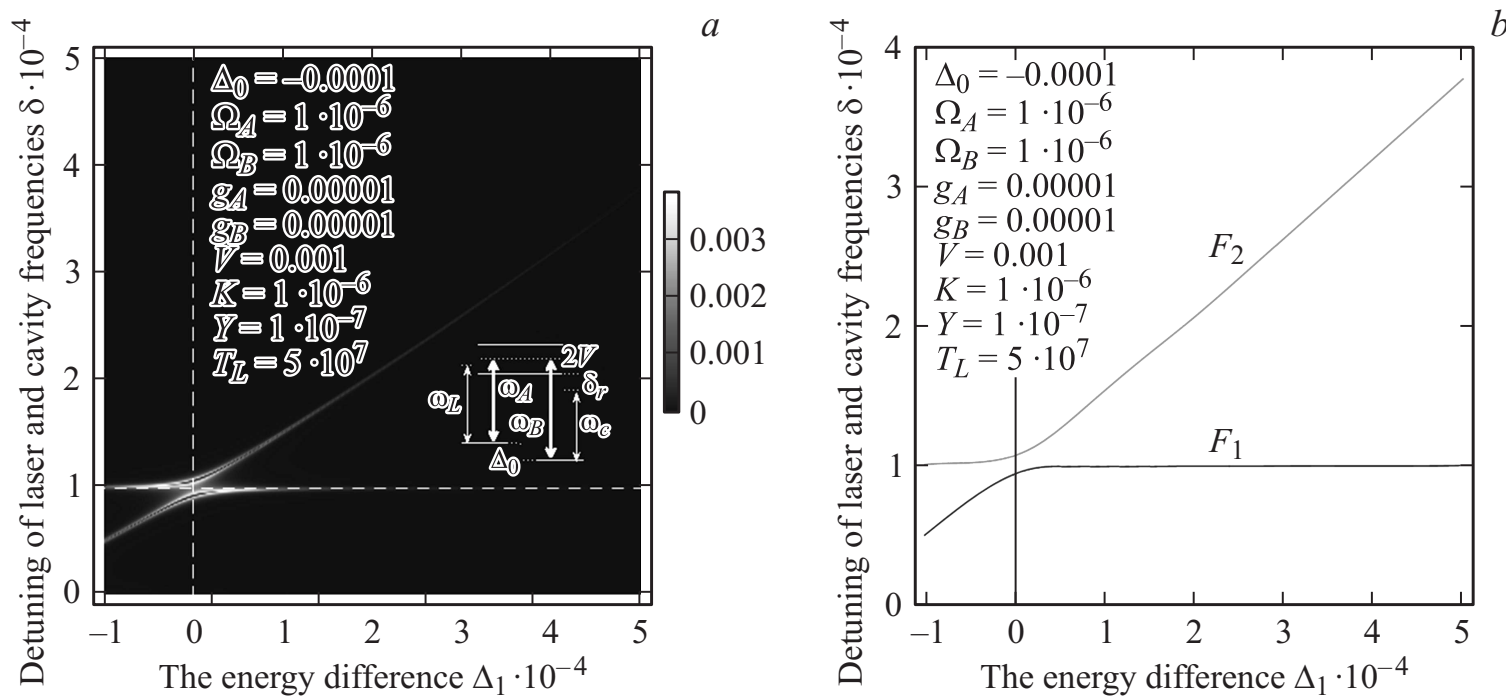

$b$

Рис. 3. $a-$ спектроскопический отклик системы в области, наиболее пригодной для реализации сенсора. $(\delta-$ разность частот резонатора и лазера, $\Delta_{1}$ - разность энергий возбужденных уровней КТ $A$ и КТ $\left.B\right) . b-$ аппроксимированные функции, соответствующие расположению максимумов $P_{\max }$ при фиксированном $\delta$.

счет создания между катодом и анодом диода разности потенциалов, квантовая система возбуждается внешним лазерным полем. В результате эксперимента анализируется прошедший (DT) и отраженный (DR) сигналы, а их частота совпадает с частотой сканирующего лазерного поля. Для реализации такой схемы потребуются достаточно сложные детекторы, которые допускают в ходе измерения осуществлять дополнительную настройку на частоту поступающего сигнала. В предложенной нами реализации данного типа приборов такая необходимость отсутствует, так как частота сигнала, попадающего на детектор, не зависит от частоты возбуждающего поля лазера, постоянна и равна частоте МР $\omega_{c}$.

Для дальнейшего исследования характеристик сенсора электрического поля на основе ДКТ необходимо аппроксимировать полученные зависимости отстройки $\delta$ от расщепления между возбужденными уровнями КТ $\Delta_{1}$ гладкими непрерывными функциями. При этом геометрическое место точек для аппроксимации этими функциями соответствует координатам $\delta$ и $\Delta_{1}$ максимумов $P_{\max }$ при фиксированном $\delta$. Результат для двух ветвей представлен на рис. $3, b$. Зависимость $\delta\left(\Delta_{1}\right)$, отвечающая кривой $F_{2}$, является важным результатом, так как именно с помощью нее и будет производиться нахождение $\Delta 1$, отвечающей измеряемому электрическому полю $|\mathbf{E}(\mathbf{r})|$ в процессе сканирования по частотам лазера $\omega_{L}$.

Чувствительность сенсора - характеристика прибоpa, показывающая насколько хорошо с его помощью возможно отследить малое изменение внешнего электрического поля. К настоящему моменту разработаны разные подходы к определению этого параметра для данного типа устройств. В рассмотренной выше работе [13] чувствительность наноэлектрометра определяется как $\eta=\varepsilon^{-1 / 2} \alpha^{-1} \frac{\sqrt{\dot{n}}}{\partial \dot{n} / \partial \Delta}$, где $\varepsilon-$ диэлектрическая проницаемость, $\alpha$ - постоянный дипольный момент КТ, $n$ - количество фотонов, попадающих на детектор за единицу времени, $\Delta$ - отстройка частот лазера и перехода между возбужденным и основным уровнями КТ. Экспериментально полученная чувствительность прибора достигает значения $\eta \sim 0.5(\mathrm{~B} / \mathrm{M}) / \sqrt{\Gamma ц}$.

В нашей работе чувствительность сенсора определяется производной $\frac{\partial \delta}{\partial \Delta_{1}}$ вдоль кривых $F_{1}$ и $F_{2}$, и соответственно наибольший интерес представляют те участки зависимости $\delta\left(\Delta_{1}\right)$ (рис. $3, b$ ), где она достигает

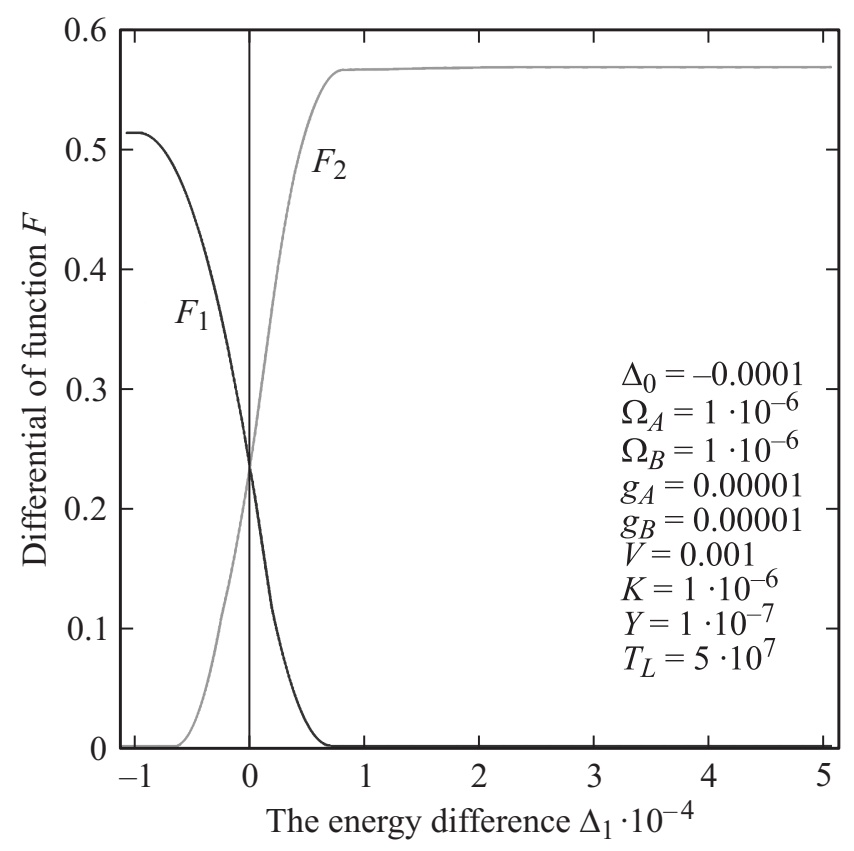

Рис. 4. Производные функций $F_{1}$ и $F_{2}$. Значение $\partial \delta / \partial \Delta_{1}$ соответствует величине чувствительности сенсора. 


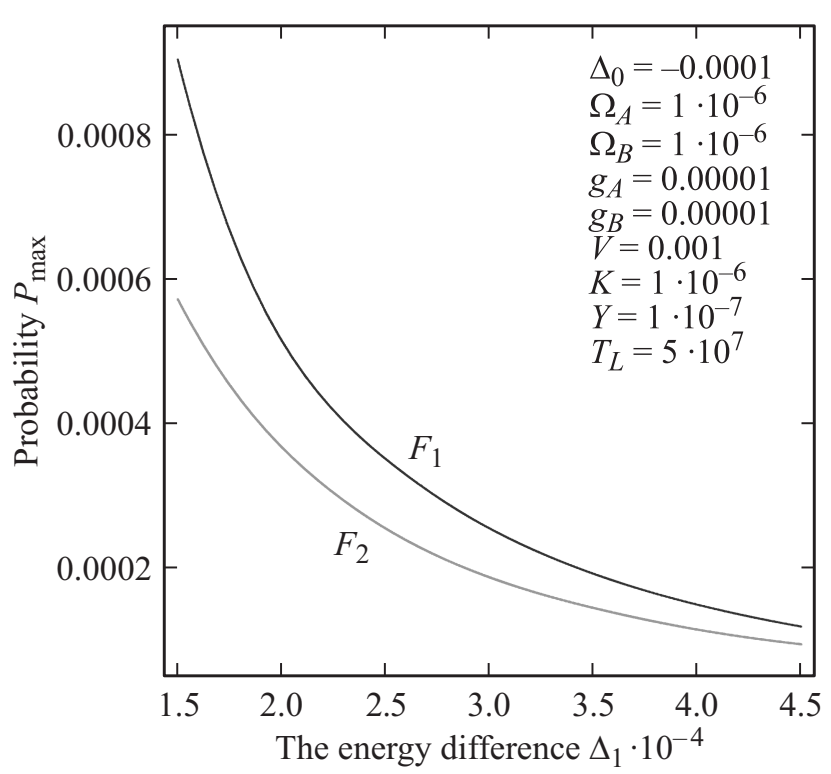

Рис. 5. Зависимость $P_{\max }$ от $\Delta_{1}$ вдоль кривых $F_{1}$ и $F_{2}$.

наибольших значений. На рис. 4 представлены полученные величины производных для кривых $F_{1}$ и $F_{2}$. Как мы можем видеть из графика, максимальное значение чувствительности достигается на кривой $F_{2}$ и стремится к величине $\eta=0.56$. При движении по кривой $F_{1}$ в сторону увеличения $\Delta_{1}$ чувствительность прибора падает до нуля, а при уменьшении $\Delta_{1}$ параметры системы стремятся к области первого антипересечения, вблизи которой пропадает селективность системы по частоте. Таким образом, для сенсорики особый интерес представляют значения параметров, лежащие на кривой $F_{2}$, и для ясности будем называть ее рабочей.

Проведем численную оценку для чувствительности $\eta=0.56$. Производная $\frac{\partial \delta}{\partial \Delta_{1}}$ связана с величиной $\frac{\partial \delta}{\partial E}$ через дипольный момент КТ как $\frac{\partial \delta}{\partial \Delta_{1}}=\frac{\partial \delta}{\partial(\vec{E}, \vec{d})}=\frac{1}{a e} \frac{\partial \delta}{\partial E}$, где $\vec{d}-$ дипольный момент КТ, $a-$ характерный размер КТ, $e-$ заряд электрона, $E=|\vec{E}|-$ напряженность внешнего электрического поля. Таким образом, получим численное значение чувствительности сенcopa $\eta=\frac{\partial \delta}{\partial E} \approx 10 \mathrm{MГц} \frac{\mathrm{M}}{\mathrm{B}}$. Эта величина соответствует значению $\alpha$ в работе [13], где $\alpha=0.028 \mathrm{MГц} \frac{\mathrm{M}}{\mathrm{B}}$, что на 4 порядка меньше, чем полученное значение для сенсора на основе ДКТ. Таким образом, предложенная нами конструкция сенсора имеет более высокую чувствительность, что позволит обнаружить слабые отклонения напряженности электрических полей. Далее исследуем шумовую устойчивость прибора. Для этого необходимо получить значения максимумов $P_{\max }$ вдоль кривых $F_{1}$ и $F_{2}$.

Как мы можем видеть (рис. 5), максимальная заселенность уровней с числом фотонов более нуля падает по мере роста расщепления между возбужденными уровнями КТ $\Delta_{1}$. В данном случае это связано с отда- лением параметров системы от области, соответствующей условию наличия двухфотонного резонанса (9), в окрестности второго антипересечения. Минимальное значение $\Delta_{1}=1.5 \cdot 10^{-4}$ выбрано особым образом, что будет показано далее.

Проанализируем влияние паразитных эффектов, возникающих в системе в ходе измерения сигнала. Они характеризуются наличием уширения у резонансного контура, что мешает определению точного положения максимума $P_{\max }$ при фиксированном значении $\Delta_{1}$. Величина шума равна уширению кривой на высоте $P_{\max } / 2$ и соответствует величинам $\Gamma_{1}$ и $\Gamma_{2}$. При стремлении к области $\Delta_{1} \approx 0$ резонансные контуры приближаются друг к другу и в области $-1.5 \cdot 10^{-4} \leq \Delta_{1} \leq 1.5 \cdot 10^{-4}$ сливаются таким образом, что невозможно отличить один максимум от другого. Будем говорить, что уширения $\Gamma_{1}$ и $\Gamma_{2}$ не определены в данной области, а график этих функций терпит разрыв. Поэтому дальнейшее исследование системы будет проделано для значений расщепления из диапазона $1.5 \cdot 10^{-4} \leq \Delta_{1} \leq 4.5 \cdot 10^{-4}$.

Уширение $Г$ падает по мере увеличения разности между возбужденными уровнями КТ $\Delta_{1}$. В первую очередь это связано с тем, что уменьшается и значение максимальной вероятность $P_{\max }$ при росте $\Delta_{1}$. Вторым фактором, влияющим на величину $\Gamma$, является постепенная отстройка параметров системы при увеличении $\Delta_{1}$ от условия двухфотонного резонанса (9), что негативно влияет на величину шумовой составляющей сигнала, поступающей на детектор. В результате этого величина Г спадает медленнее, чем $P_{\max }$, и приводит к следующему графику соотношения сигнал/шум в системе (рис. 6).

Как можно видеть из полученной зависимости, соотношение сигнал/шум достигает своего максимального значения при $\Delta_{1}=1.5 \cdot 10^{-4}$. На участке

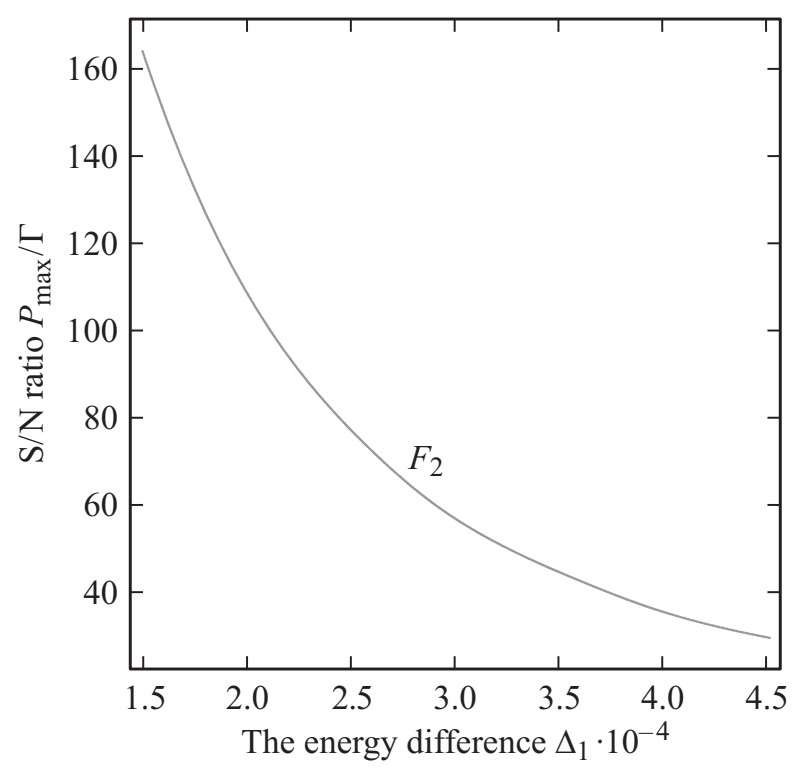

Рис. 6. Соотношение сигнал/шум (равное $\left.P_{\max } / \Gamma\right)$ для сенсора электрического поля на основе ДКТ в зависимости от $\Delta_{1}$ для кривой $F_{2}$. 
$1.5 \cdot 10^{-4} \leq \Delta_{1} \leq 4.5 \cdot 10^{-4}$ для рабочей кривой $F_{2}$ оно падает с 165 до 30, что составляет 5.5 раза.

Произведем некоторые оценки для диапазона полей, измеряемого с помощью сенсора на основе ДКТ, рассмотренного в данной работе. Расщепление возбужденных уровней ДКТ связано с величиной внешнего поля через дипольный момент КТ следующим образом: $\Delta_{1}=$ ae $\vec{E} \vec{d}$, где $a-$ характерный размер КТ, $\vec{E}-$ вектор внешнего электрического поля, $\vec{d}-$ единичный вектор дипольного момента КТ. Проведем оценку для KT, выполненной из GaAs (численные значения параметров приведены в разд. 3) с характерным размером $a=10 \mathrm{Hм}: \quad E=\Delta_{1} / a e ; \quad 1.5 \cdot 10^{10} \Gamma_{ц} \leq \Delta_{1} \leq 5 \cdot 10^{10} \Gamma_{ц ;}$ $900 \mathrm{~B} / \mathrm{M} \leq E \leq 3000 \mathrm{~B} / \mathrm{M}$.

\section{5. Заключение}

Разработана схема сенсора электрического поля на основе ДКТ, помещенной в МР и управляемой полем лазера. Рассчитан спектроскопический отклик системы и определен диапазон параметров, пригодных для реализации сенсора. Исследована шумовая устойчивость прибора и указан интервал амплитуд полей, которые можно измерить с его помощью. Показано, что устройство обладает рядом конкурентных преимуществ по сравнению с аналогами, а именно высокой чувствительностью, достигающей $\eta=\frac{\partial \delta}{\partial E} \approx 10 \mathrm{MГц} \frac{\mathrm{M}}{\mathrm{B}}$, селективностью системы по частоте, что позволяет использовать различные каналы для возбуждения и измерения, возможностью за счет малых размеров $(a \sim 10$ нм $)$ получать пространственное распределения электрического поля с точностью $\eta_{n} \sim 50 \mathrm{Hм}^{2}$, а также простотой реализации и готовой технической базой.

Работа поддержана Программой фундаментальных научных исследований ОНИТ РАН ,Элементная база квантовых компьютеров“ (проект 1.5).

\section{Список литературы}

[1] R. Freeman, I. Willner. Chem. Soc. Rev., 41, 4067 (2012).

[2] C. Schneider, T. Heindel, A. Huggenberger, P. Weinmann, C. Kistner, M. Kamp, S. Reitzenstein, S. Höfling, A. Forchel. Appl. Phys. Lett., 94, 111111 (2009).

[3] А.В. Цуканов, В.Г. Чекмачев. Микроэлектроника, 45, 1 (2016).

[4] А.В. Цуканов, И.Ю. Катеев. Микроэлектроника, 43, 323 (2014).

[5] А.В. Цуканов, И.Ю. Катеев. Микроэлектроника, 43, 403 (2014).

[6] А.В. Цуканов, И.Ю. Катеев. Микроэлектроника, 44, 79 (2015).

[7] C. Dory, A.F. Kevin, K. Müller, K.G. Lagoudakis, T. Sarmiento, A. Rundquist, J.L. Zhang, Y. Kelaita, J. Vučković. Scientific Reports, 6, 25172 (2016).

[8] A. Barenco, C.H. Bennett, R. Cleve, D.P. DiVincenzo, N. Margolus, P. Shor, T. Sleator, J.A. Smolin, H. Weinfurter. Phys. Rev. A, 52, 3457 (1995).
[9] Л.А. Опенов, А.В. Цуканов. ФТП, 38, 94 (2004).

[10] G.W. Wen, J.Y. Lin, H.X. Jiang, Z. Chen. Phys. Rev. B, 52 5913 (1995).

[11] K. Srinivasan, O. Painter. Nature, 450, 862 (2007).

[12] M. Brandstetter, M.A. Kainz, T. Zederbauer, M. Krall, S. Schönhuber, H. Detz, W. Schrenk, A.M. Andrews, G. Strasser, K. Unterrainer. Appl. Phys. Lett., 108, 011109 (2016).

[13] A.N. Vamivakas, Y. Zhao, S. Fält, A. Badolato, J.M. Taylor, M. Atatüre. Phys. Rev. Lett., 107, 166802 (2011).

Редактор А.Н. Смирнов

\section{Stark-shift based quantum dot-cavity electrometer}

\author{
A.V. Tsukanov ${ }^{1,2}$, V.G. Chekmachev ${ }^{1,2}$ \\ ${ }^{1}$ Moscow Institute of Physics and Technology, \\ 141701 Dolgoprudny, Russia \\ 2 Institute of Physics and Technology, \\ Russian Academy of Sciences, \\ 117218 Moscow, Russia
}

\begin{abstract}
In this paper we propose a scheme of an optical quantum sensor of external electric field based on a double quantum dot which placed in a high- $Q$ semiconductor microcavity. The model of dynamic processes occurring in the system is developed, the spectral characteristics of the device are investigate as well the noise stability of the sensor is studied. It is shown that, thanks special design, such a device has some advantages such as high sensitivity, the existence of different channels for the excitation and measurement, the ability accurately determine the spatial distribution of the electric field.
\end{abstract}

